\title{
Development of novel low temperature bonding technologies for microchip chemical analysis applications
}

\author{
A. Sayah*, D. Solignac, T. Cueni, M.A.M. Gijs \\ Institute of Microsystems, Swiss Federal Institute of Technology Lausanne, CH-1015 Lausanne EPFL, Switzerland
}

Received 9 August 1999; received in revised form 28 October 1999; accepted 1 November 1999

\begin{abstract}
We introduce two new low temperature bonding technologies for the assembly of microstructured glass substrates for the realisation of microchannels for miniaturised chemical analysis applications. A first method consists of a proper cleaning of the two glass surfaces, followed by a simple epoxy gluing process at $90^{\circ} \mathrm{C}$. In a second method, direct bonding is obtained just by exposing the glass stack to a high pressure (up to $50 \mathrm{MPa}$ ) in the $100-200^{\circ} \mathrm{C}$ temperature range. We obtain bonding strengths as high as $10 \mathrm{MPa}$, higher than the best values obtained by HF-assisted or plasma-assisted bonding. For the realisation of the microchannels, we introduce, besides the well-known HF-etching technology, two simple alternative methods, namely, sawing and micropowder blasting. (C) 2000 Elsevier Science S.A. All rights reserved.
\end{abstract}

Keywords: Bonding; Sandblasting; Pressure; Low temperature

\section{Introduction}

The availability of a low temperature and reliable wafer bonding technology is essential for many microsystems applications [1], ranging from the fabrication of siliconon-insulator wafers [2], silicon-glass wafer bonding for microsensor packaging schemes [3] to the realisation of all glass microchips for miniaturised chemical analysis applications [4]. It is well known that large bonding strengths can be obtained when exposing the wafers to a high temperature treatment; for example, for silicon fusion bonding, temperatures above $1000^{\circ} \mathrm{C}$ need to be applied [2], while for Pyrex glass fusion bonding, temperatures of the order of $600^{\circ} \mathrm{C}$ are necessary [5]. However, the use of high temperature procedures can be detrimental for previous micropatterning sequences of the wafers, as well can prevent the use of certain metals in the bonding stack. Therefore, field-assisted silicon to glass bonding (anodic bonding) techniques have been developed, allowing strong $\mathrm{Si}$-glass bonds at temperatures lower than $450^{\circ} \mathrm{C}$. Recently, several new developments of true low temperature bonding processes $\left(100-200^{\circ} \mathrm{C}\right)$ have been reported. These

\footnotetext{
* Corresponding author. Tel.: +41-21-693-6583; fax: +41-21-6935950.

E-mail address: abdeljalil.sayah@epfl.ch (A. Sayah)
}

comprise the use of an intermediate layer of sodium silicate solution between the two wafers to be bonded [6,7] and exposure in a vacuum system of the wafer surface to plasma and ion beam treatment to activate bonding surfaces $[8,9]$. A very interesting recent development is the low temperature pressure-assisted bonding of two $\mathrm{SiO}_{2}$ wafers using a wafer surface treatment by dilute hydrofluoric acid (HF 0.2-1 wt.\%) for quartz microchip analysis applications [10]. Bonding strengths as high as $6 \mathrm{MPa}$ were obtained at temperatures below $100^{\circ} \mathrm{C}$. However, caution should be taken when working with HF solution in the hot press system.

In this paper, we propose the use of direct pressure-assisted low temperature bonding for assembling two microstructured glass substrates (soda-lime microscope glass slides), without the use of HF. A first method consists of a proper cleaning of the two glass surfaces, the deposition of a tiny $(1 \mu \mathrm{m})$ epoxy gluing layer, followed by the application of an external pressure $(1 \mathrm{MPa})$ at $90^{\circ} \mathrm{C}$; in a second method, direct bonding is obtained just by exposing the cleaned glass substrates to a high pressure (up to $50 \mathrm{MPa}$ ) in the $100-200^{\circ} \mathrm{C}$ temperature range. We obtain bonding strengths as high as $10 \mathrm{MPa}$, higher than the best values obtained by HF-assisted or plasma-assisted bonding. We utilise our techniques for the realisation of microchannels for electrophoresis applications, by bonding one unstruc- 
tured and one micropatterned glass substrate. For the micropatterning of these channels, we propose, besides the well known HF etching technology, two new methods: sawing and micropowder blasting.

\section{Microchips for electrophoresis}

The integration of chemical analysis systems on a microchip is a research area of increasing importance today $[4,11]$. The miniaturisation is motivated by the possibility to use extremely small amounts of sample to be analysed, to increase the speed of analysis, and to allow massive parallelism in analysis sequences. Up to now, various miniaturised separation techniques have been realised on microchips, such as capillary electrophoresis [12,13], gas chromatography [14] and high-pressure liquid chromatography [15]. A common feature of the liquid-based analysis systems is the use of a small microchannel for sample injection and a longer one for separation. Due to its good electrical isolation and good optical transparency, glass is one of the favourite materials used in the fabrication of separation microsystems. Fig. 1 is an optical photograph of the capillary electrophoresis chip we used in our experiments; it consists of a standard cross-channel geometry. In all cases, ordinary soda-lime glass microscope slides (76 $\times 26 \times 1 \mathrm{~mm}^{3}$ ) were used as starting substrates. For realising the cross-channel micropattern, we have used three different methods, as described below.

\section{Microchannel fabrication}

Prior to the bonding of the two glass slides, a channel structure needs to be patterned on one of the substrates. For this purpose, we have used three different techniques: wet etching using $\mathrm{HF}$, sawing and powder-blasting. The second substrate is provided with access holes, made by powder blasting, for the insertion of liquids and electrophoresis driving electrodes. In the case of HF-etching, the most frequently used technique [5], a metal mask layer is realised by a lift-off technique. A total of $2.0-\mu \mathrm{m}$ positive resist (AZ 5214) is patterned photolithographi-

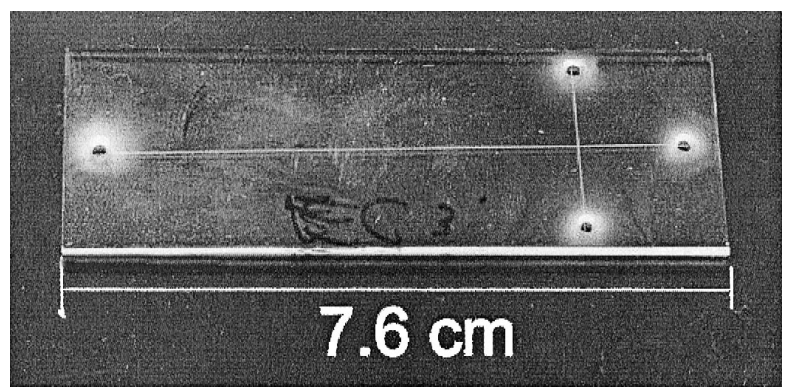

Fig. 1. Optical photograph of the capillary electrophoresis chip; it consists of a standard cross-channel geometry.

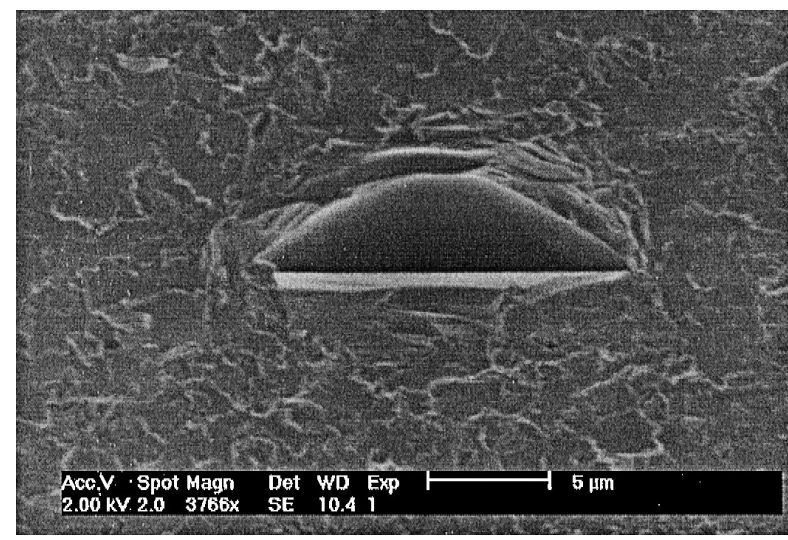

Fig. 2. SEM photograph of the cross-section of two bonded substrates showing isotropic profile of the HF etched channel.

cally; subsequently, a metal layer consisting of $150 \AA$ chromium and $2000 \AA$ gold is evaporated on the substrate. Next, the photoresist is removed by acetone, leading to the inverse image of the cross-channel mask. Wet chemical etchingwith HF solution (49\%) is used to etch the glass substrate; the etching rate at room temperature is about 7 $\mu \mathrm{m} / \mathrm{min}$. Since the HF etching process is isotropic; the etched channels obtained have a width two times larger than the etched depth, hence, only channels with semi-elliptical cross-section are possible. Fig. 2 is a scanning electron microscope (SEM) photograph and represents a typical example of the cross-section of two bonded substrates with an isotropic profile of the HF etched channel.

Aiming for a simplification of the channel micropatterning, we have used, in a second technique, a sawing machine (KS 775 type wafer saw), where we have adjusted the sawing depth to control the depth of the channel. The width of the channel is simply determined by the thickness of the saw blade. The minimum industrially available saw blade thickness is about $30 \mu \mathrm{m}$. Fig. 3 shows an example of the channel cross-section obtained with this technique; the saw blade thickness used in this case is $300 \mu \mathrm{m}$. This technique has the advantage to be simple, fast, no mask is

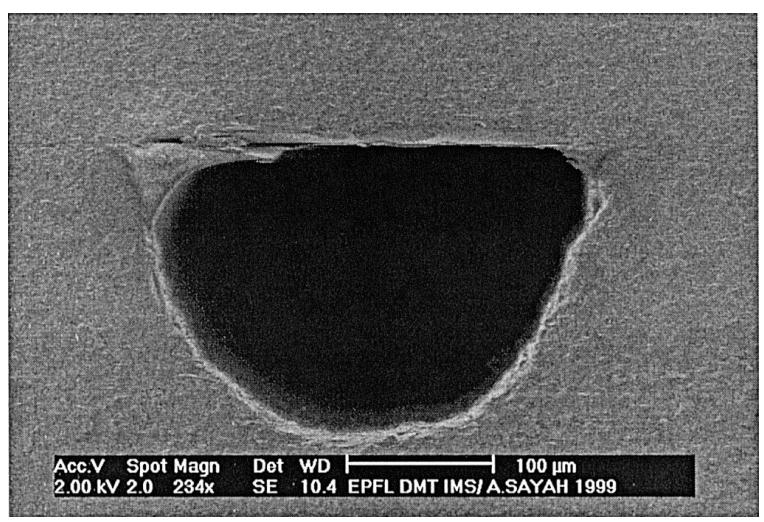

Fig. 3. SEM photograph of the channel cross-section obtained by the sawing technique; the saw blade thickness is $300 \mu \mathrm{m}$. 


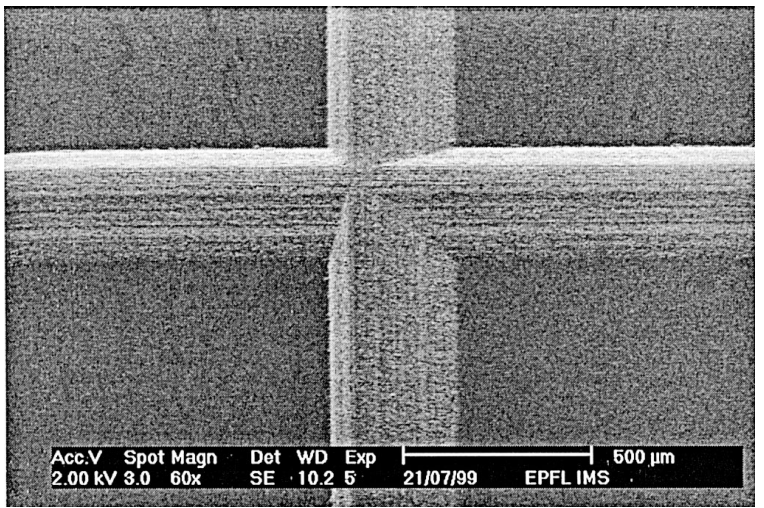

Fig. 4. SEM photograph of the crossing point of the sample injection and separation channels obtained by the sawing technique.

necessary and there is no need of clean room environment. Moreover, more circular-like channels can be realised, which often is advantageous for chemical separation. However, a disadvantage of the method is the dependence of the channel shape on the quality of the saw blade; another disadvantage is the limitation in design freedom of the micropatterned channels, as only straight channels are possible using this method. A photograph of the crossingpoint of the sample injection and separation channels is shown in Fig. 4.

The third technique we have used for the microchannel patterning is powder blasting. We recently have introduced this method as a new technique for the fabrication of microsystems: a high pressure powder blasting jet with particle velocities of the order of $200 \mathrm{~m} / \mathrm{s}$ is directed toward the glass substrate, covered by a $500 \mu \mathrm{m}$ thick metal mask. Under the impact of this eroding powder jet, cracks are created, leading to the removal of the glass. The characteristics of this technique and the experimental set-up are described in great detail elsewhere [16]. Fabrication of microsystems with this technique necessitates the use of a masking material with high wear resistance, like a metal, photoresist or plastics. The minimum obtainable dimension

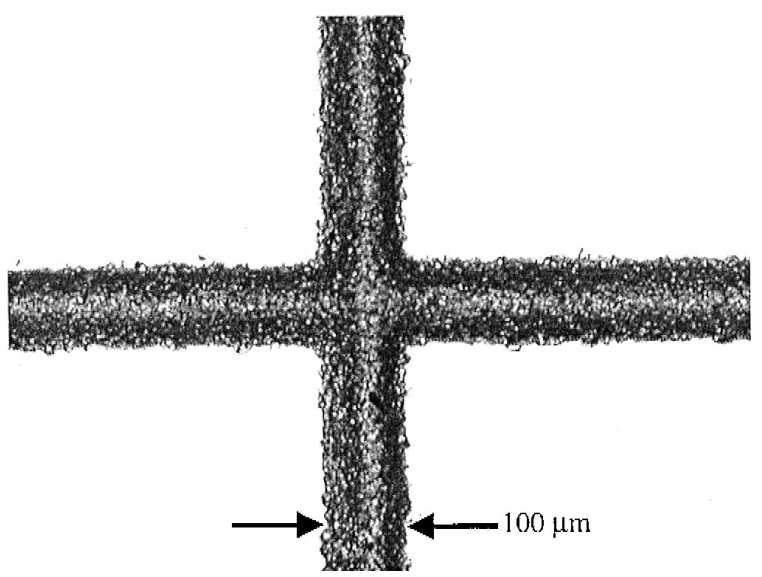

Fig. 5. Optical photograph of the cross channel geometry, as obtained by powder blasting, the channels surface is very rough.

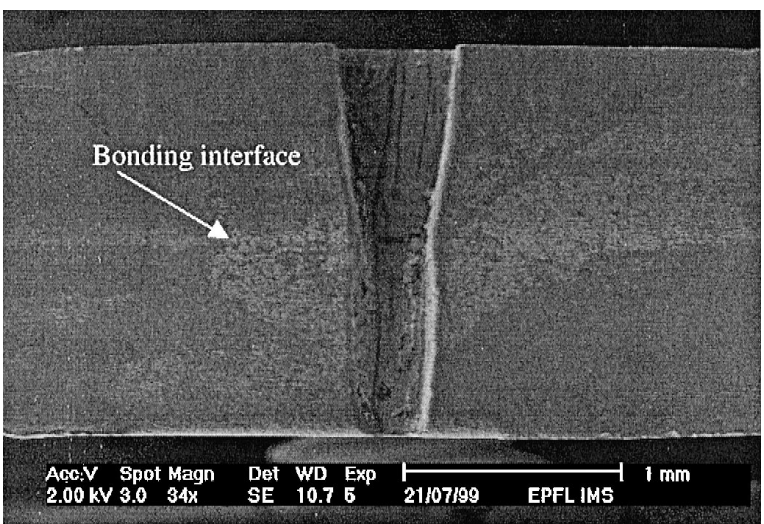

Fig. 6. SEM photograph of a cross sectional view of a hole, eroded through two bonded glass substrates; the anisotropic etching profile and the high aspect ratio of the hole structure are clearly visible.

of the patterned structure is essentially limited by the size of the powder particles; for example, with particles of about $30-\mu \mathrm{m}$ size the minimum patterning resolution that can be obtained is around $50 \mu \mathrm{m}$. The etching rate of the glass substrate is about $100 \mu \mathrm{m} / \mathrm{min}$, which is very fast compared to the etching rates obtained by wet and dry etching. In addition, the powder blasting technique allows high flexibility in design of microchannel patterns, the realisation of anisotropic etching profiles and high aspect ratio structures. Fig. 5 shows the cross-channel geometry, as obtained by powder blasting. The surface of the channels is relatively rough, which may be an advantage for the adhesion of stationary phases in chromatographic applications. Moreover, due to the very high etching rate, powder blasting is very suitable for the fabrication of access holes, forming the interconnection of the microchannel to the external reservoirs. In Fig. 6, we can see a cross-sectional view of a hole, eroded through two bonded glass substrates; the anisotropic etching profile and the high aspect ratio of the hole structure are clearly visible.

\section{Bonding technologies}

The bonding process consists of several steps: we always start with the preparation of the wafers by a chemical treatment of their surface. Direct wafer bonding depends enormously on the chemical surface state of the wafers to be bonded, since intermolecular or interatomic forces play a major role in obtaining a good bonding strength. Therefore, wafer surfaces must be sufficiently clean, flat, and smooth. First, the substrates are cleaned in an organic solvent to remove particles, dust and organic contamination. Then, a sulphuric acid peroxide mixture $\left(\mathrm{H}_{2} \mathrm{SO}_{4}\right.$ : $\left.\mathrm{H}_{2} \mathrm{O}_{2}=5: 1\right)$ is used to increase the hydrophilicity of the glass substrates. This step is carried out at room temperature for $20 \mathrm{~min}$. Due to the high viscosity of the sulphuric acid, afterwards, wafers are intensively rinsed in deionised 
water and dried. The next steps in the bonding process depend on the choice of the bonding method as described below.

\subsection{Fusion bonding}

High temperature fusion bonding is the technique of reference for comparing with our low temperature bonding processes. For soda-lime glass substrates, the fusion bonding is carried out at $600^{\circ} \mathrm{C}$, close to the melting temperature. At this high temperature, there is a nearly perfect bonding of the two surfaces, as shown in Fig. 7: indeed, with this technique, no interface between the two wafers is visible. To evaluate the bonding strength, we mounted the samples into a pull test machine (Autotrac DY-30, ADAMEL LHOMARGY). The bonded wafer was diced into test samples. All the bonded wafers withstood the dicing process, which can be considered as the preliminarily evaluation of the bonding strength. Two steel supports are glued to either side of the bonded sample and mounted in the test machine. The force required for breaking the sample is measured; from this, we derive values for the bonding strength of about $20-30 \mathrm{MPa}$, close to the bond-
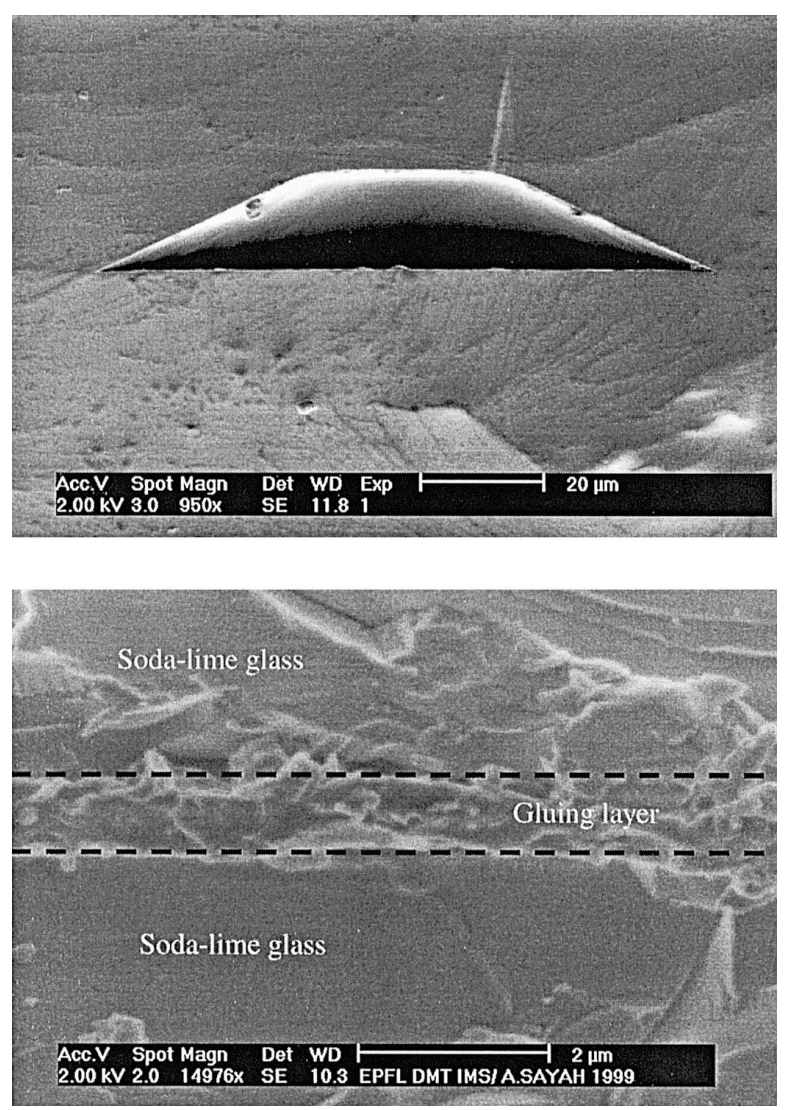

Fig. 7. SEM photograph of a microchannel structure realised by fusion bonding; no interface is visible.

Fig. 8. SEM photograph of a cross-section of the bonded substrates with the $1-\mu \mathrm{m}$ thick intermediate gluing layer.

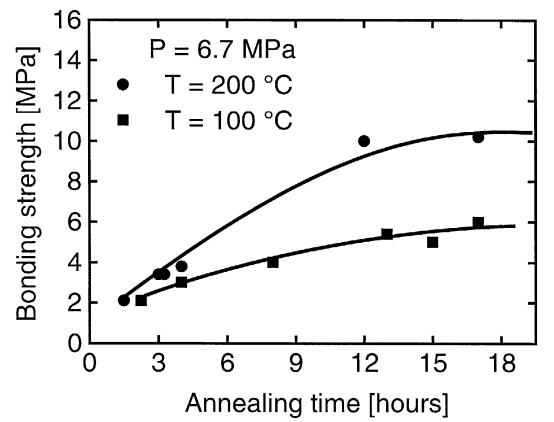

Fig. 9. Variation of the bonding strength vs. the bonding time at a constant pressure of $6.7 \mathrm{MPa}$ and for temperatures of $100^{\circ} \mathrm{C}$ and $200^{\circ} \mathrm{C}$. The solid curves are a guide for the eye.

ing strength of the bulk (44 MPa, measured using the same method).

\subsection{Gluing}

A first simple technique to realise bonding at low temperature is the use of an intermediate gluing layer. Two component epoxy resin (Araldit) has been chosen for gluing the two substrates. A potential problem with this method is the ability of the glue to block the channels. To avoid this problem, we used the following process: before bonding, a glass substrate with a $1-\mu \mathrm{m}$ thick layer of a glue, is heated at $80^{\circ} \mathrm{C}$ until the glue starts to harden. At the moment, the glue is still soft enough to bond well and its viscosity is sufficiently high to prevent filling of the channel, the second glass substrate is brought into contact with the first one. A pressure of about $1 \mathrm{MPa}$ is applied during hardening of the glue, permitting a uniform bonding. A cross-section of the bonded substrates with the $1-\mu \mathrm{m}$ thick intermediate gluing layer is shown in Fig. 8. A clear advantage of this method is its simplicity and very low temperature; however, a potential drawback is the interference of the hardened epoxy material with the chemical analysis experiments. Therefore, we focused our attention to the direct pressure-assisted bonding as discussed in Section 4.3.

\subsection{Pressure-assisted low temperature bonding}

The pressure-assisted low temperature bonding was carried out with a CARVER 4120 press, allowing to apply pressures up to $50 \mathrm{MPa}$ at temperatures up to $340^{\circ} \mathrm{C}$. The bonding process is as follows.

(1) Immediately after cleaning, the two substrates are brought into contact and a variable external pressure is applied to the whole surface of the substrates pair. This applied pressure presents two advantages: first, it permits to keep the two substrates sufficiently close to allow the intermolecular forces to be effective. Second, it produces a small deformation of the substrate surfaces, enabling the 


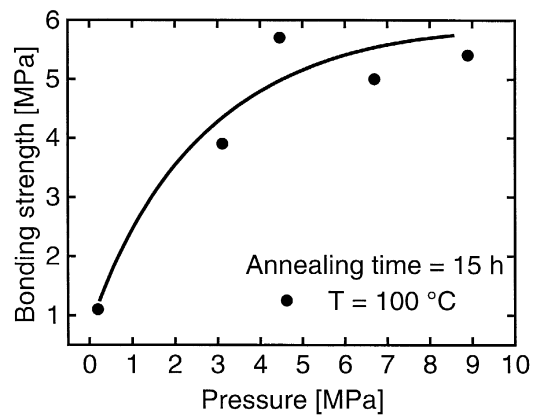

Fig. 10. The influence of the applied pressure on the bonding strength for an annealing time of $15 \mathrm{~h}$ and a temperature of $100^{\circ} \mathrm{C}$.

elimination of voids in between the two substrates, originating from surface imperfections and roughness.

(2) While continuously maintaining the applied pressure, the substrate stack is annealed at a specific temperature.

We have studied the bonding strength in function of the various experimental parameters: pressure, temperature and bonding time. Fig. 9 presents the variation of the bonding strength vs. the bonding time at a constant pressure of 6.7 $\mathrm{MPa}$ and for temperatures of $100^{\circ} \mathrm{C}$ and $200^{\circ} \mathrm{C}$. The bonding strength increases with bonding time until it reaches a constant value around 6 and $10 \mathrm{MPa}$ at an annealing temperature of $100^{\circ} \mathrm{C}$ and $200^{\circ} \mathrm{C}$, respectively, which is sufficiently high for capillary electrophoresis applications. For both annealing temperatures, saturation bonding strengths are already reached after $12 \mathrm{~h}$ of the annealing time. The chemical mechanism behind the bonding process is governed by the polymerisation of the $\mathrm{Si}-\mathrm{OH}$ groups on the pre-bonded substrates into strong equivalent $\mathrm{Si}-\mathrm{O}-\mathrm{Si}$ bonds under the release of $\mathrm{H}_{2} \mathrm{O}$ [17]. The effect of the pressure on the bonding strength is merely the contact surface enhancement between the two substrates, thereby changing the defective area where bonding will occur. The influence of the applied pressure on the bonding strength for an annealing time of $15 \mathrm{~h}$ and a temperature of $100^{\circ} \mathrm{C}$ is shown in Fig. 10. We observe that the bonding strength increases with the applied pressure until it reaches a constant value around $6 \mathrm{MPa}$. After the bonding strength test, the appearance of the interface of the fractured substrate stack was observed with an optical microscope. This inspection reveals the following:

1. In most cases, this interface contains two parts: one corresponding to the sample broken in the bulk and the other to the interface between the two bonded substrates;

2. The portion of the part broken in the bulk increases essentially with the pressure applied during the bonding.

Based on these observations, the relation between the pressure and the bonding strength indeed may be attributed to the flatness of the substrate surfaces. In fact, parts of the two substrates in close proximity show a strong bonding, whereas parts with small intersubstrate gaps have a weak bonding. The applied pressure probably produces an elastic deformation of the substrates, thereby reducing the number of gaps and increasing the effectively measured bonding strength.

\section{Conclusions}

We have introduced direct pressure-assisted low temperature bonding for assembling two microstructured soda-lime microscope glass slides for chemical analysis microchip applications, without the use of HF or plasma activation of the surfaces. A first method consists of a proper cleaning of the two glass surfaces, the deposition of a $1-\mu \mathrm{m}$ thick epoxy gluing layer, pre-hardening of the glue, followed by the application of a small external pressure $(1 \mathrm{MPa})$ at $90^{\circ} \mathrm{C}$. This method results in hermetically sealed channels, but interference of epoxy material with chemical analysis procedures favour our second low temperature method. Here, direct bonding is obtained just by exposing the cleaned glass substrates to a high pressure (up to $50 \mathrm{MPa}$ ) in the $100-200^{\circ} \mathrm{C}$ temperature range. We obtain bonding strengths as high as $10 \mathrm{MPa}$, well above the best values obtained by HF-assisted or plasma-assisted bonding. We realised microchannels for electrophoresis applications, by bonding one unstructured and one micropatterned glass substrate. For the micropatterning of these channels, we proposed, besides the well-known HF etching technology, two new methods, namely, sawing and micropowder blasting.

\section{References}

[1] M.A. Schmidt, Wafer-to-wafer bonding for microstructure formation, Proc. IEEE 86 (1998) 1575.

[2] W.P. Maszara, Silicon-on-insulator by wafer bonding: a review, J. Electrochem. Soc. 138 (1991) 341.

[3] H. Hemmi, S. Shoji, Y. Shoji, M. Esashi, Vacuum packaging for microsensors by glass silicon anodic bonding, Sens. Actuators, A 43 (1994) 243.

[4] A. Manz, N. Graber, H.M. Widmer, Miniaturised total analysis systems: a novel concept for chemical sensing, Sens. Actuators, B 1 (1990) 244

[5] D.J. Harrison, K. Fluri, K. Seiler, Z. Fan, C.S. Effenhauser, A. Manz, Micromachining a miniaturized capillary electrophoresisbased chemical analysis system on a chip, Science 261 (1993) 895.

[6] R. Puers, A. Cozma, Bonding wafers with sodium silicate solution, J. Micromech. Microeng. 7 (1997) 114.

[7] A. Satoh, Water glass bonding, Sens. Actuators, A 72 (1999) 160.

[8] Y.A. Li, R.W. Bower, Systematic low temperature silicon bonding using pressure and temperature, Jpn. J. Appl. Phys. 37 (1998) 737.

[9] H. Takagi, R. Maeda, N. Hosoda, T. Suga, Integration of dissimilar materials room-temperature wafer bonding using Ar beam surface activation, Proc. Transducers 99, Sendai, Japan (1999) 1328. 
[10] H. Nakanishi, T. Nishimoto, M. Kanai, T. Saitoh, K. Nakamura, S. Shoji, Condition optimisation, reliability evaluation of $\mathrm{SiO}_{2}-\mathrm{SiO}_{2}$ HF bonding and its application for UV detection micro flow cell, Proc. Transducers 99, Sendai, Japan (1999) 1332.

[11] D.J. Harrison, A. van den Berg (Eds.), Micro Total Analysis Systems '98, Kluwer Academic Publishers, Dordrecht, 1998.

[12] S.C. Jacobson, A.W. Moore, J.M. Ramsey, Fused quartz substrates for microchip electrophoresis, Anal. Chem. 67 (1995) 2063.

[13] Z.H. Fan, D.J. Harisson, Micromachining of capillary electrophoresis injectors and separators on glass chips and evaluation of flow at capillary intersections, Anal. Chem. 66 (1994) 177.

[14] S.C. Terry, J.H. Jerman, J.B. Angell, A gas chromatographic air analyzer fabricated on a silicon wafer, IEEE Trans. Electron Devices ED 26 (1979) 1880.

[15] A. Manz, Y. Miyahara, J. Miura, Y. Watanaba, H. Miyagi, K. Sato, Design of an open-tubular column liquid chromatograph using silicon chip technology, Sens. Actuators, B 1 (1990) 249.

[16] E. Belloy, S. Thurre, E. Walckiers, A. Sayah, M.A.M. Gijs, Powder blasting as a new technology for microsystems fabrication, Sens. Actuators, A, submitted.

[17] Q.Y. Tong, T.H. Lee, U. Gosele, The role of surface chemistry in bonding of standard silicon wafers, J. Electrochem. Soc. 144 (1997) 384.

\section{Biographies}

Abdeljalil Sayah received his degree in Physics in 1988 from University Mohammed V, Rabat, specialising in state solid physics. He obtained his AESA and DEA in microelectronics specialising in the technology of the components in 1990 and 1992, respectively. From 1993 until 1996, he worked as a Research Assistant in the CNET-Bagneux (Paris) Laboratory where he received a $\mathrm{PhD}$ degree for the thesis entitled "Realisation of Silicon-based dielectrics Optical Waveguide on InP by Photochemical Deposition'. In 1996, he joined the Applied Optics Institute (IOA) of the Swiss Federal Institute of Technology in Lausanne (EPFL) as a postdoctoral research. His research interests included the study and characterisation of optical fiber tips for scanning near field optical microscopy and participated on the characterisation of Bragg grating produced in optical fibers by UV laser. He is now with the Institute for Microsystems (IMS) at EPFL. His current research interests are in inertial sensor (gyroscope), biosensors, and fabrication technologies.
Dominique Solignac was born in Lodeve, France, in 1973. He receiced the post-graduate Diploma in heterochemical, polymers and catalysis in 1996 from the University of Sciences and Technics in Montpellier. After a 1-year training research period in organic synthesis at the University of Lausanne, he joined in May 1999, the Swiss Federal Institute of Technology, EPF Lausanne as a Research Assistant. His research interest are in microfabrication technologies for microfluidics and microanalysis systems.

Martin A.M. Gijs received his degree in Physics in 1981 from the Katholieke Universiteit Leuven, Belgium and his PhD degree in Physics at the same university in 1986. He joined the Philips Research Laboratories in Eindhoven, The Netherlands, in 1987. Subsequently, he has worked there on micro- and nanofabrication processes of high critical temperature superconducting Josephson and tunnel junctions, the microfabrication of microstructures in magnetic multilayers showing the giant magnetoresistance effect, the design and realisation of miniaturised motors for hard disk applications and the design and realisation of planar transformers for miniaturised power applications. He joined the Swiss Federal Institute of Technology Lausanne (Ecole Polytechnique Fédérale de Lausanne) in 1997 as a Professor in the Institute of Microsystems of the Microengineering Department, where he is responsable for the Microsystems Technology group. His main interests are in developing technologies for novel inductive-type devices, new microfabrication technologies for microsystems fabrication in general and the development and use of microsystems technologies for biomedical applications in particular.

T. Cueni is a graduate student in Microengineering, who contributed to the present work during the course of a student research project. 